(C) 1984. The Genetical Society of Great Britain

\title{
SELF-INCOMPATIBILITY IN RYEGRASS VIII. THE MODE OF ACTION OF $S$ AND $Z$ ALLELES IN THE POLLEN OF AUTOTETRAPLOIDS OF LOLIUM PERENNE L.
}

\author{
C. H. FEARON*, M. D. HAYWARD † AND M. J. LAWRENCE* \\ *Department of Genetics, University of Birmingham, Birmingham B15 2TT. + Welsh Plant \\ Breeding Station, Plas Gogerddan, Aberystwyth SY23 3EB
}

Received 16.xii.83

\section{SUMMARY}

Though autotetraploid ryegrasses are as self-incompatible as the diploids from which they have been induced, hitherto it has not been known for certain whether only one $S-Z$ pair in the pollen of tetraploids must be matched by the same pair in the stigma for incompatibility to occur (hypothesis 1) or whether all such pairs have to be matched (hypothesis 2). Having determined, in the previous investigation, the incompatibility genotypes of the members of two families of autotetraploid ryegrass (Fearon et al., 1984), it is now possible to decide which of these hypotheses is true by using pollen from each of these tetraploids on the stigmas of related diploids of known genotype.

The results of this part of the investigation of the inheritance and expression of self-incompatibility in autotetraploid ryegrass show with very little doubt that the first hypothesis is true; that is, only one $S-Z$ pair of the pollen of tetraploids needs to be matched in the stigma for incompatibility to occur.

The effects of both aneuploidy and of double reduction on our ability to distinguish between the pattern of pollinations expected on each hypothesis are considered; it is shown that neither of these disturbances is likely to reduce our ability to discriminate between the two hypotheses.

These results thus confirm those of Lundqvist (1957, 1962), which were obtained from investigations on autotetraploid rye and Festuca pratensis.

\section{INTRODUCTION}

In the preceding paper we described a three-part procedure designed to elucidate the genetics of incompatibility in autotetraploid ryegrass which involves the use of four full-sib families, the members of each being related to those of the others as half-sibs. Two of these families were produced by crossing diploid ryegrasses $\left(26^{2} \times 23^{2}\right.$ and $\left.23^{2} \times 1^{2}\right)$ and the other two by crossing tetraploid plants $\left(26^{4} \times 1^{4}\right.$ and its reciprocal). The results obtained from the first part of this procedure, which involved the use of pollen of diploids of known incompatibility genotype on the stigmas of their related tetraploids, revealed that the 35 legitimate tetraploid plants examined fell into 18 different incompatibility classes and that the alleles at each incompatibility locus act independently in the stigmas of tetraploids. The gametic segregation ratios obtained were consistent with the assumption that the chromosomes assort at random at meiosis in the parents of these tetraploids (Fearon, Hayward and Lawrence, 1984).

The second part of this procedure, considered here, involves the use of pollen of tetraploids on the stigmas of their related diploids, the purpose of which is to elucidate the mode of action of the incompatibility alleles in the pollen of tetraploids, about which two contrasting hypotheses exist. 
Thus, either only one $S-Z$ pair in the pollen needs to be matched in the stigma for incompatibility to occur (hypothesis 1); or, alternatively, all of the $S-Z$ pairs in the pollen, of which there can be as many as four, must be matched in the stigma for incompatibility to occur (hypothesis 2 ).

\section{Materials AND METHODS}

The genotypes of the parents of the families with which we are concerned are, from the results of the first experiment, as follows:

$$
\begin{array}{cc}
\text { Cross } & \text { Genotypes } \\
26^{2} \times 23^{2} & S_{34} Z_{34} \times S_{12} Z_{12} \\
23^{2} \times 1^{2} & S_{12} Z_{12} \times S_{56} Z_{56} \\
26^{4} \times 1^{4} & S_{3344} Z_{3344} \times S_{5566} Z_{5566} \\
1^{4} \times 26^{4} & S_{5566} Z_{5566} \times S_{3344} Z_{3344}
\end{array}
$$

The genotypes of the factorial set of four testers from diploid family $26^{2} \times 23^{2}$ are:

$$
S_{-3} Z_{-3} ; S_{-3} Z_{-4} ; S_{-4} Z_{-3} \text { and } S_{-4} Z_{-4}
$$

the dash subscript indicating that the identity of the first allele at each locus is irrelevant to our present purpose. Similarly, the genotypes of the factorial set of four testers from the diploid family $23^{2} \times 1^{2}$ are:

$$
S_{-5} Z_{-5} ; S_{-5} Z_{-6} ; S_{-6} Z_{-5} \text { and } S_{-6} Z_{-6}
$$

A total of 49 tetraploids were examined in the first experiment, of which 35 were legitimate and 14 the open-pollinated progeny of the two parents. Two of the legitimate tetraploids failed to survive the winter between the first and second experiment and six others emerged from winter in too poor a condition to flower. One of these losses was the sole representative of its incompatibility class $\left(S_{3456} Z_{3455}\right)$ and two others were the only representatives of theirs $\left(S_{3455} Z_{3456}\right)$, so that only 16 classes occurred among the survivors. Each of the 14 open-pollinated progeny survived the winter, which, with the 27 legitimate plants, gave 41 tetraploid plants in all.

Further details of materials and methods are given in Fearon et al., (loc. cit.) and Scarrott (1981).

\section{THEORY}

Three genotypic classes can be recognised among the tetraploids in respect of the alleles they have inherited from parent $26^{4}$ :

\section{Class}

Double heteroallelic

Single heteroallelic

Double homoallelic
Genotype

$$
\begin{aligned}
& S_{34-\ldots} Z_{34--} \\
& S_{33--} Z_{34--} ; S_{44-.} Z_{34--} ; \text { etc. }
\end{aligned}
$$

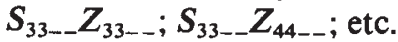

The proportion of pollen produced by the plants of each of these classes that can be recognised by the stigmas of the diploid testers from family $26^{2} \times 23^{2}$ on each of the hypotheses under test is shown in table 1 . The fractions shown in this table indicate the proportion of pollen of each of the nine tetraploid genotypes that is expected to be incompatible on the 
TABLE 1

Proportion of pollen produced by each tetraploid genotypic class as distinguished with respect to the alleles inherited from parent $26^{4}\left(\mathrm{~S}_{3344} \mathrm{Z}_{3344}\right)$, that can be recognised by the stigmas of the testers from diploid family $26^{2} \times 23^{2}\left(\mathrm{~S}_{-3} \mathrm{Z}_{-3} ; \mathrm{S}_{-3} \mathrm{Z}_{-4} ; \mathrm{S}_{-4} \mathrm{Z}_{-3} ; \mathrm{S}_{-4} \mathrm{Z}_{-4}\right)$ on the assumption of random chromosome assortment

\begin{tabular}{|c|c|c|c|c|c|c|c|c|c|}
\hline \multirow[b]{3}{*}{ Class } & \multirow[b]{3}{*}{$\operatorname{sssszzZZ}$} & \multicolumn{8}{|c|}{ Proportion of pollen of indicated genotype relevant to: } \\
\hline & & \multicolumn{4}{|c|}{ Hypothesis 1} & \multicolumn{4}{|c|}{ Hypothesis 2} \\
\hline & & $S_{3-} Z_{3-}$ & $S_{3-} Z_{4}$ & $S_{4} Z_{3-}$ & $S_{4-} Z_{4-}$ & $S_{33} Z_{33}$ & $S_{33} Z_{44}$ & $S_{44} Z_{33}$ & $S_{44} Z_{44}$ \\
\hline $\begin{array}{l}\text { Double } \\
\text { heteroallelic }\end{array}$ & $34--34--$ & $\frac{1}{4}$ & $\frac{1}{4}$ & $\frac{1}{4}$ & $\frac{1}{4}$ & - & - & - & - \\
\hline $\begin{array}{l}\text { Single } \\
\text { heteroallelic }\end{array}$ & $\begin{array}{l}33--34-- \\
34--33-- \\
34--44-- \\
44--34--\end{array}$ & $\begin{array}{l}\frac{5}{12} \\
\frac{5}{12} \\
\\
x\end{array}$ & $\frac{\frac{5}{12}}{\frac{5}{12}}$ & $\frac{\frac{5}{12}}{\frac{5}{12}}$ & $\begin{array}{l}- \\
\frac{5}{12} \\
\frac{5}{12}\end{array}$ & $\begin{array}{l}- \\
- \\
-\end{array}$ & $\begin{array}{l}- \\
-\end{array}$ & $\begin{array}{l}- \\
- \\
-\end{array}$ & $\begin{array}{l}- \\
- \\
-\end{array}$ \\
\hline $\begin{array}{l}\text { Doube } \\
\text { homoallelic }\end{array}$ & $\begin{array}{l}33--33-- \\
33--44-- \\
44--33-- \\
44--44--\end{array}$ & $\frac{\frac{25}{36}}{-}$ & $\frac{\overline{25}}{\frac{25}{36}}$ & $\frac{1}{\frac{25}{36}}$ & $\frac{-}{\frac{25}{36}}$ & $\frac{\frac{1}{36}}{-}$ & $\frac{\overline{\frac{1}{36}}}{-}$ & $\frac{-}{\frac{1}{36}}$ & $\frac{-}{\frac{1}{\frac{1}{36}}}$ \\
\hline
\end{tabular}

TABLE 2

The pattern of pollinations expected when pollen from each of the tetraploid genotypes of families $26^{4} \times 1^{4}$ and $1^{4} \times 26^{4}$ is applied to the stigmas of the four testers from $26^{2} \times 23^{2}$. The fractions indicate the proportion of compatible pollen expected on the assumption of random chromosome assortment and no dominance. Key: $+=$ fully compatible

\begin{tabular}{|c|c|c|c|c|c|c|c|c|c|c|}
\hline & $S$ & 3 & 3 & 3 & 3 & 4 & 3 & 3 & 4 & 4 \\
\hline & $S$ & 4 & 3 & 4 & 4 & 4 & 3 & 3 & 4 & 4 \\
\hline & $S$ & - & - & - & - & - & - & - & - & - \\
\hline & $S$ & - & - & - & - & - & - & - & - & - \\
\hline & $Z$ & 3 & 3 & 3 & 4 & 3 & 3 & 4 & 3 & 4 \\
\hline & $Z$ & 4 & 4 & 3 & 4 & 4 & 3 & 4 & 3 & 4 \\
\hline & $Z$ & - & - & - & - & - & - & - & - & - \\
\hline & $Z$ & - & - & - & - & - & - & - & - & - \\
\hline \multicolumn{11}{|l|}{$S S Z Z$} \\
\hline$-3-3$ & & $\frac{3}{4}$ & $\frac{7}{12}$ & $\frac{7}{12}$ & + & + & $\frac{11}{36}$ & + & + & + \\
\hline$-3-4$ & Hvo 1 & $\frac{3}{4}$ & $\frac{7}{12}$ & + & $\frac{7}{12}$ & + & + & $\frac{11}{36}$ & + & + \\
\hline$-4-3$ & пур. 1 & $\frac{3}{4}$ & + & $\frac{7}{12}$ & + & $\frac{7}{12}$ & + & + & $\frac{11}{36}$ & + \\
\hline$-4-4$ & & $\frac{3}{4}$ & + & + & $\frac{7}{12}$ & $\frac{2}{12}$ & + & + & + & $\frac{11}{36}$ \\
\hline$-3-3$ & & + & + & + & + & + & $\frac{35}{36}$ & + & + & + \\
\hline$-3-4$ & Hun 2 & + & + & + & + & + & + & $\frac{35}{36}$ & + & + \\
\hline$-4-3$ & Нyр. 2 & + & + & + & + & + & + & + & $\frac{35}{36}$ & + \\
\hline$-4-4$ & & + & + & + & + & + & + & + & + & $\frac{35}{36}$ \\
\hline
\end{tabular}

stigmas of each of the four diploid testers on each hypothesis; hence one minus this fraction gives the expected compatibility of the cross (table 2 ). For example, consider the cross $S_{13} Z_{13} \times S_{3456} Z_{3456}$, which in terms of the alleles inherited from parents $26^{2}$ and $26^{4}$, respectively, is $S_{-3} Z_{-3} \times S_{34 \ldots-} Z_{34 \ldots-.}$. Assuming random chromosome assortment, one quarter of the pollen produced by a tetraploid of this genotype will carry $S_{3}$ and $Z_{3}$ and none will be $S_{33} Z_{33}$. Hence this cross is expected to be three-quarters compatible on 
hypothesis 1 , but fully compatible on hypothesis 2 . The remaining expectations in table 2 are obtained in a similar way. A corresponding set of expectations with respect to the alleles inherited from parent $1^{4}\left(S_{5}, S_{6}, Z_{5}\right.$ and $Z_{6}$ ) may be obtained in the same fashion.

It is clear from table 2 that the pattern of pollinations between the tetraploids and diploid testers expected on hypothesis 1 is very different from that expected on hypothesis 2, for if the latter is true, all but four of the 36 pollinations are expected to be fully compatible. Furthermore, since in practice, it will be virtually impossible to distinguish between a pollination which is $\frac{35}{36}$ compatible and one that is fully compatible, the expectation on hypothesis 2 is that all of the crosses between the diploid testers and the tetraploids will appear to be fully compatible. With hypothesis 1 , on the other hand, 16 of the 36 pollinations are expected to be partially compatible, 4 of which are expected to be three-quarters compatible, with the rest appearing to be half-compatible, since in practice it will be difficult to distinguish pollinations which are $\frac{11}{36}$ from those which are $\frac{7}{12}$ compatible.

\section{Results}

The results obtained by pollinating at least one plant of each of the four tester genotypes from each diploid family with pollen from each of the 41 tetraploids are shown in figs. 1 and 2 . Of the 408 pollinations carried out, 58 per cent were partially compatible, clearly indicating that only one $S-Z$ pair in the pollen of tetraploids needs to be matched in the stigma for incompatibility to occur (hypothesis 1). In classifying the pollinations, of the 156 expected on this hypothesis to be three-quarters compatible, 153 were scored as $\mathrm{T}$, two as + and one as $\mathrm{H}$. Again, of the 75 pollinations that are expected to be $\frac{7}{12}$ compatible, 63 were scored as $\mathrm{H}$ and 12 as $\mathrm{T}$. All of the pollinations that are expected to be $\frac{11}{36}$ compatible have been scored as $\mathrm{H}$. The incidence of misclassification in the present data is thus very close to that encountered when handling families of diploids (Cornish, Hayward and Lawrence, 1979; Fearon, Hayward and Lawrence, 1983, 1984).

\section{Discussion}

The expectations shown in table 2 were derived on the assumption that all pollen from tetraploids is diploid; and that, at meiosis, there is random chromosome assortment. Both of these assumptions appear to be reasonable, since it has been shown that each of the 41 tetraploids involved in the present investigation had the expected number of 28 chromosomes and that the gametic segregation ratios of their parents were consistent with the hypothesis of random chromosome assortment (Fearon et al., 1984). On the other hand, very many more pollen grains can be observed on each stigma in an average pollination than the number of plants examined. It is possible, therefore, that events which cannot be detected among zygotes may be detectable in the pollen. Furthermore, since quadrivalents occur at meiosis in these tetraploids, double reduction is clearly possible (Scarrott, 1981). Lastly, the observation that all plants in the experiment are euploid cannot be taken as evidence that all gametes are also euploid, for the disadvantages of aneuploidy may be largely confined to zygotes. What, 


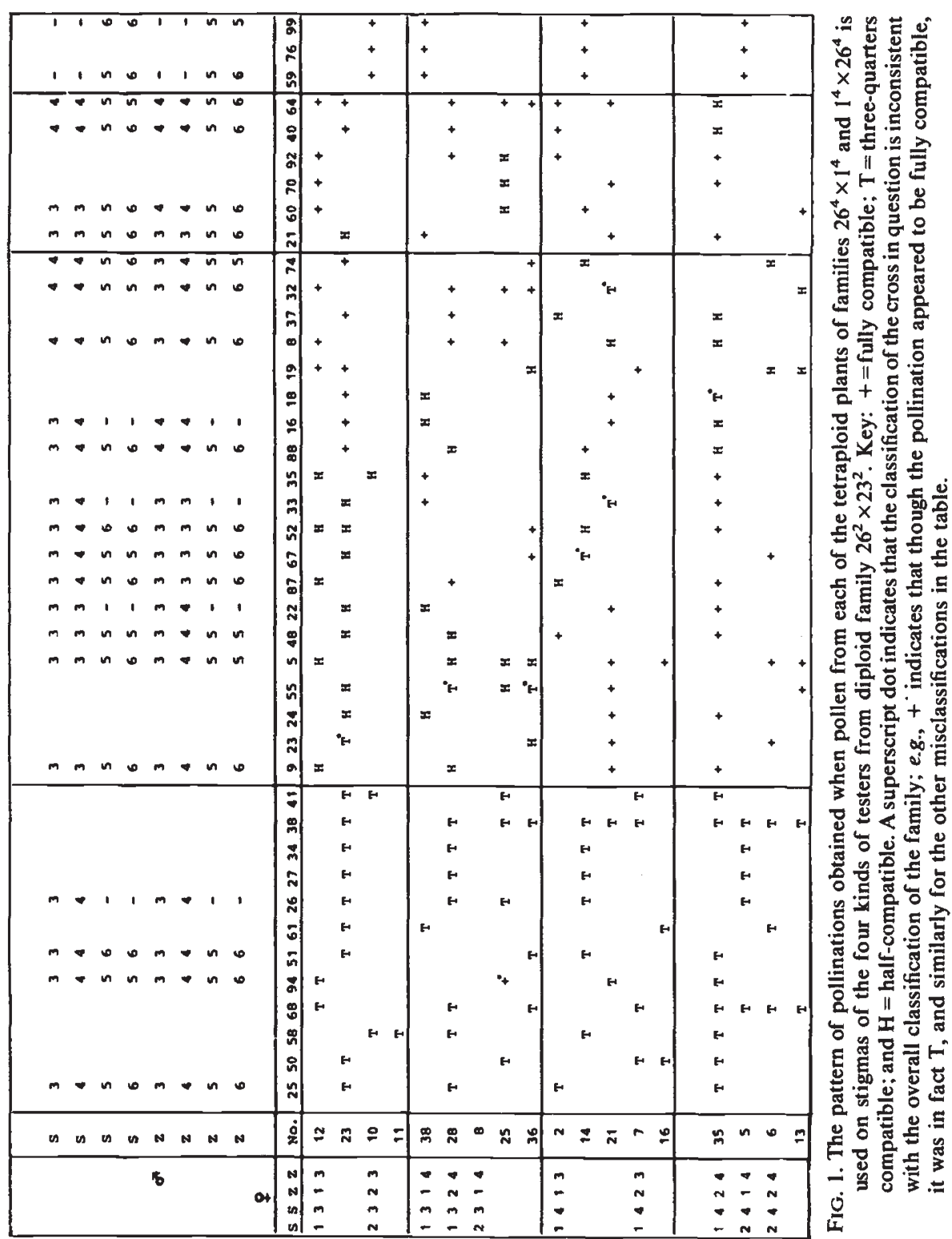




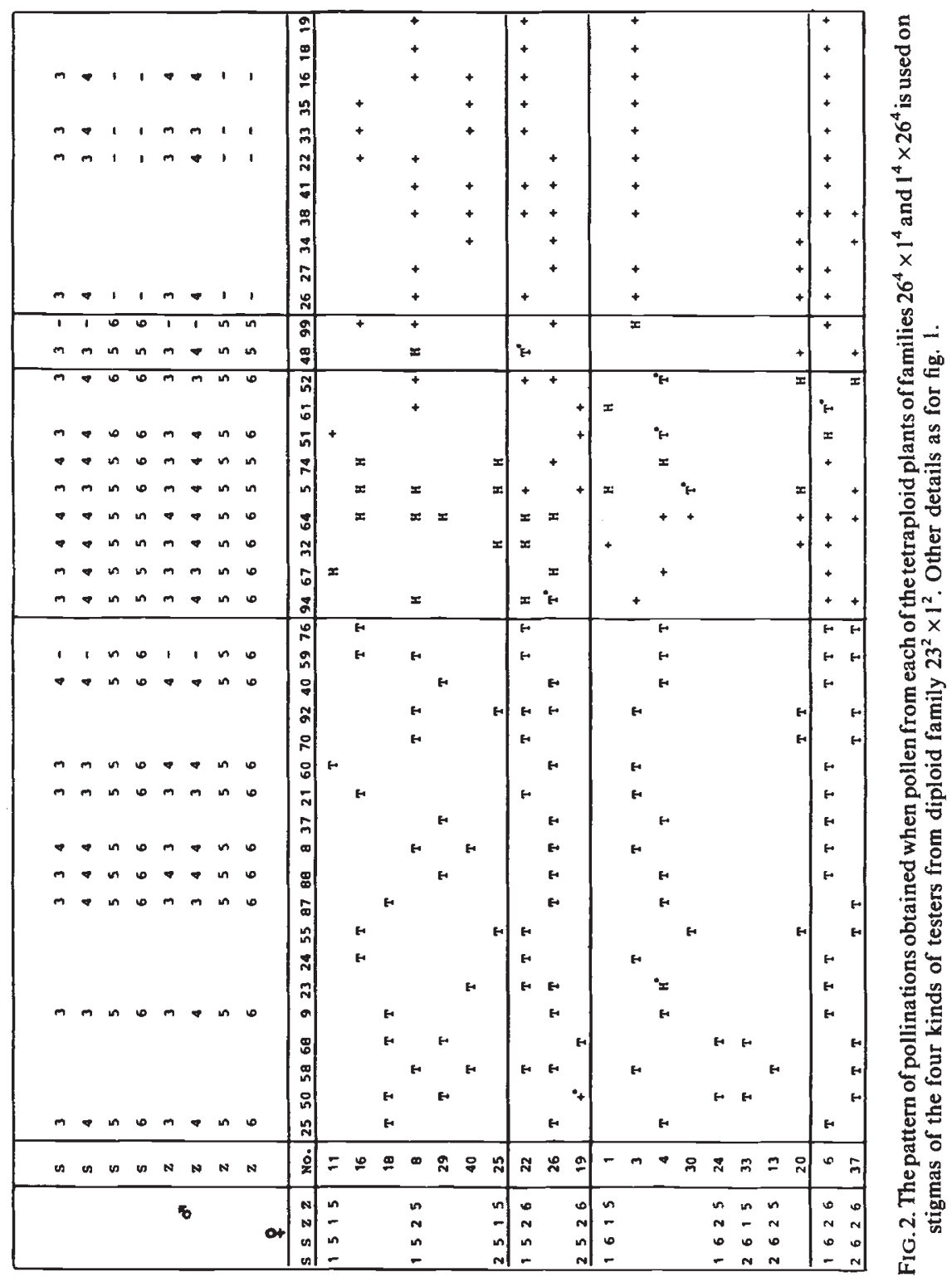


then, are the effects of aneuploidy and of double reduction on the expectations shown in table 2 and to what extent can these effects reduce our ability to discriminate between the hypotheses under test?

The effect of aneuploidy on these expectations depends partly on whether the incompatibility genes are carried on the same chromosome. Though the evidence on this question is equivocal, it is nevertheless possible that $Z$, as well as $S$, is carried on chromosome 6 of $L$. perenne (Cornish, Hayward and Lawrence, 1980; Fearon, et al., 1983). Consider, then, a cross of the type $S_{-3} Z_{-3} \times S_{34--} Z_{34--}$ which, it will be recalled, is expected to be threequarters compatible on the first and fully compatible on the second hypothesis. If $S$ and $Z$ are linked, loss of the chromosome bearing $S_{3}$ and $Z_{3}$ during gamete production will cause an increase in the level of compatibility expected on hypothesis 1 , while the outcome expected on hypothesis 2 remains unaffected. Alternatively if loss occurs such that $S_{3}$ and $Z_{3}$ are the only incompatibility alleles which remain in the pollen, such pollen grains will be incompatible on both hypotheses; that is, the level of compatibility expected on hypothesis 2 will be reduced. These situations may also arise if $S$ and $Z$ are unlinked and the pollen is reduced in respect of both the $S$ - and the $Z$-bearing chromosomes, but this seems improbable. Again, assuming there is no linkage between $S$ and $Z$, if a chromosome bearing $S_{3}$ or $Z_{3}$ is lost, the proportion of gametes carrying $S_{3} Z_{3}$ will be reduced so that the level of compatibility expected on hypothesis 1 will be increased while that expected on hypothesis 2 will remain unaffected. Alternatively, if an incompatibility allele is lost such that $S_{3}\left(\right.$ or $\left.Z_{3}\right)$ alone remains in the pollen together with two $Z$ (or two $S$ ) alleles, then the results predicted on either hypothesis are not affected.

The effect of trisomic pollen on the expectations for this cross is opposite to that of monosomic pollen. In tetraploid ryegrasses, however, the frequency of monosomic pollen appears to be approximately twice that of trisomic pollen (Evans and Davies, 1982; Simonsen, 1973). Overall, therefore, the effect of aneuploidy on the expectations shown in table 2 is to increase the degree of compatibility expected on hypothesis 1 and reduce that expected on hypothesis 2 . However, it is doubtful whether any such changes would be sufficiently large to be detected in practice. Similar conclusions are arrived at when the other kinds of tetraploid pollinators are considered (i.e., single heteroallelics and double homoallelics). Hence the possibility that aneuploidy could seriously reduce our ability to discriminate between these hypotheses can be ruled out.

The effect of double reduction on the expectations shown in table 2 may be investigated by assuming that $S$ and $Z$ segregate independently and that for each locus, the proportion $(\alpha)$ of gametes which are doubly reduced is $\frac{1}{6}$. Double reduction will occur at this high frequency only in the unlikely event of the appropriate chromosomes always forming quadrivalents and there always being one chiasma between the locus and the centromere. Thereafter, random disjunction of the quadrivalent is assumed (see Mather, 1935). The modified expectations (table 3) show that the overall effect of double reduction is to increase the degree of compatibility expected on hypothesis 1 and to decrease that expected on hypothesis 2 . However, it is again very doubtful whether this slight reduction in the magnitude of the difference between these two sets of expectations could be detected in practice. In short, the possibility that double reduction, either on its own 
TABLE 3

The effect of double reduction on the expectations of table 2. The compatibility of each cross on each hypothesis is shown for $\alpha=0$ and $\alpha=\frac{1}{6}$ at each incompatibility locus in fractional (above) and decimal form (below)

\begin{tabular}{ccccccc}
\hline & \multicolumn{2}{c}{ Hypothesis 1} & & \multicolumn{2}{c}{ Hypothesis 2} \\
\cline { 2 - 3 } \cline { 5 - 6 } Cross & $\alpha=0$ & $\alpha=\frac{1}{6}$ & & $\alpha=0$ & $\alpha=\frac{1}{6}$ \\
\hline$S_{-3} Z_{-3} \times S_{34--} Z_{34--}$ & $\frac{3}{4}$ & $\frac{455}{576}$ & & 1 & $\frac{575}{576}$ \\
$S_{-3} Z_{-3} \times S_{33--} Z_{34--}$ & $(0.750)$ & $(0.790)$ & & $(1.000)$ & $(0.998)$ \\
& $\frac{7}{12}$ & $\frac{139}{216}$ & & 1 & $\frac{214}{216}$ \\
$S_{-3} Z_{-3} \times S_{33--} Z_{33--}$ & $(0.583)$ & $(0.644)$ & & $(1.000)$ & $(0.991)$ \\
& $\frac{11}{36}$ & $\frac{32}{81}$ & & $\frac{35}{36}$ & $\frac{77}{81}$ \\
& $(0.310)$ & $(0.395)$ & & $(0.972)$ & $(0.951)$ \\
\hline
\end{tabular}

or in conjunction with aneuploidy, could seriously reduce our ability to discriminate between the two hypotheses can be discounted; hence the results can be taken at their face value.

The conclusion that only one $S-Z$ pair of alleles in the pollen of tetraploid ryegrass needs to be matched by the same pair in the stigma for incompatibility to occur thus confirms that of Lundqvist $(1957,1962)$ which was drawn from his work on autotetraploid rye and Festuca pratensis. This conclusion, therefore, is likely to hold for tetraploids of all self-incompatible grasses and not just for those of the genera Secale, Festuca and Lolium.

Acknowledgements. We wish to acknowledge the award of a Science and Engineering Research Council CASE studentship to C.H.F. and an A.R.C. research grant to M.J.L. which enabled this work to be carried out.

\section{REFERENCES}

CORNISH, M. A., HAYWARD, M. D. AND LAWRENCE, M. J. 1979. Self-incompatibility in ryegrass. I. Genetic control in diploid Lolium perenne L. Heredity, 43, 95-106.

CORNISH, M. A., HAYWARD, M. D. AND LAWRENCE, M. J. 1980. Self-incompatibility in ryegrass. III. The joint segregation of $S$ and PGI-2 in Lolium perenne L. Heredity, 44, 55-62.

EVANS, G. M. AND DAVIES, E. W. 1982. The dynamics of aneuploidy in an induced tetraploid population of Lolium multiflorum $\times$ Lolium perenne. Theor. Appl. Genet. 62, 39-44.

FEARON, C. H., HAYWARD, M. D. AND LAWRENCE, M. $J$. 1983. Self-incompatibility in ryegrass. V. Genetic control, linkage and seed set in diploid Lolium multiflorum Lam. Heredity, 50, 35-45.

FEARON, C. H., HAYWARD, M. D. AND LAWRENCE, M. J. 1984. Self-incompatibility in ryegrass. VII. The determination of incompatibility genotypes in autotetraploid families of Lolium perenne L. Heredity 53, 403-413.

LUNDQVIST, A. 1957. Self-incompatibility in rye. II. Genetic control in the tetraploid. Hereditas, 43, 467-511.

LUNDQVIST, A. 1962. The nature of the two-loci incompatibility system in grasses. I. The hypothesis of a duplicative origin. Hereditas, 48, 152-168.

MATHER, K. 1935. Reductional and equational separation of the chromosomes in bivalents and multivalents. Journal of Genetics, 30, 53-78.

SCARROTT, C. H. 1981. Self-incompatibility in Diploid and Tetraploid Lolium Species. Ph.D. thesis, University of Birmingham.

SIMONSEN, Q. 1973. Cytogenetic investigation in a diploid and autotetraploid population of 5, 157-188. 